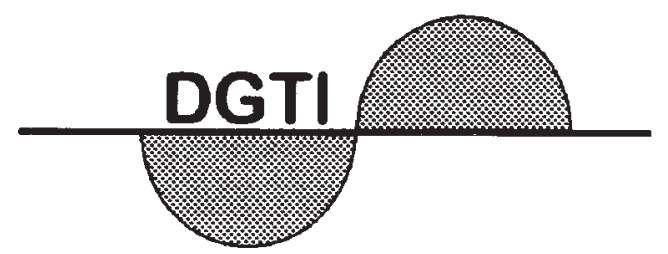

\section{Deutsche Gesellschaft für Transfusionsmedizin und Immunhämatologie}

Geschäftsstelle: Prof. Dr. Reinhold Eckstein, Abteilung für Transfusionsmedizin und Hämostaseologie, Universitätsklinikum Erlangen/Nürnberg, Krankenhausstraße 12, D-91054 Erlangen Tel. +49 $913185-6346$, Fax -6987

\section{Stellungnahme der Sektionen «Sicherheit in der Hämotherapie» und "Präparative und therapeutische Hämapherese" an den Vorstand der DGTI}

Anlässlich der gemeinsamen Tagung der DGTI-Sektionen «Sicherheit in der Hämotherapie» und «Präparative und therapeutische Hämapherese» am 3. März 2004 in Leipzig wurde über das Thema «kritische Spenderwanderung» sowohl im Hinblick auf den Spenderschutz als auch im Hinblick auf den Produktschutz diskutiert. Derzeit lässt sich aber keine genaue Aussage darüber treffen, inwieweit risikoreiche Spenderwanderungen bestehen, weil es keine diesbezüglichen Untersuchungen gibt. Die Spendeeinrichtungen haben keine Möglichkeiten zur gegenseitigen Information etabliert, da dies bislang nicht als erforderlich angesehen wurde. Verschärfter Wettbewerb unter den Spendeeinrichtungen und die Entschädigung der Blutspender werden beobachtet. Die meisten Spendeeinrichtungen akzeptieren die Angaben der Spender im Rahmen des gegenseitigen Vertrauensgrundsatzes jedoch als zuverlässig.

Von Sektionsmitgliedern wurde jedoch darauf hingewiesen, dass sich im «Entwurf zum 1. Gesetz zur Änderung des TFG» bereits ein Passus zum Datenschutz bei Einrichtung eines Spenderinformationssystems findet. In $\S 6$ Abs. 2 heißt es dort: «Das gilt entsprechend auch für Angaben über die spendende Person, die im Rahmen eines Systems der Spendeeinrichtungen zur Information über den letzten Spendetermin und die Rückstellung von spendenden Personen bereitgestellt werden». Im Kommentar zu $\$ 6$ steht: «Sie (die Spender) haben dann die Möglichkeit, dies zu akzeptieren oder von der Spendeentnahme zurückzutreten». Darüber hinaus fordern Behörden in einzelnen Bundesländern (wie z.B. in Thüringen) bereits jetzt von Spendeeinrichtungen eine Vernetzung und Meldepflicht zur Überwachung der «kritischen Spenderwanderung».

Als Transfusionsmediziner sehen wir eine solche Entwicklung mit Sorge. Ohne eine vorherige Klärung des tatsächlichen Bedarfs für ein solches Informationssystem wird bereits eine gesetzliche Grundlage zu dessen Einrichtung geschaffen. Nach unserer Auffassung ist ein solches System weder konform mit den Datenschutzauflagen, welche die EU-Richtlinie 2002/ 98/EG für Spenderdaten vorsieht, noch ist dieses Vorgehen der Spenderwerbung besonders zuträglich. Der Datenschutz für Spenderdaten darf nicht auf bloßen Verdacht hin ausgesetzt werden

Bislang liegen in Deutschland keine aktuellen Daten zur Thematik «kritische Spenderwanderung / risikoreiches Spenderverhalten» vor. Eine anonymisierte Datenerhebung zur Spenderwanderung wäre jedoch mit relativ einfachen technischen Mitteln durchführbar: Herr Dr. Fabian (Suhl) stellte bei der Sektionstagung ein Konzept vor, wie sich gleich bleibende Spendermerkmale (Vorname, Name usw.) mit einem Einbahnstraßen-Algorithmus zu einer Prüfsumme kodieren lassen, die keine Rückschlüsse auf die Original-Spenderdaten zulässt. Dabei wird Wert gelegt auf die Nutzung vorhandener EDV sowie Kosten sparender Technologien. Dieser Weg sollte zuallererst geprüft werden, natürlich vorbehaltlich einer Stellungnahme des Bundesdatenschutzbeauftragten zur generellen Zulässigkeit. Ohne das Vorliegen einer solchen Datenerhebung ist eine medizinische Notwendigkeit für die Vernetzung und Meldung von Spenderdaten nicht gegeben und die kostenintensive Einrichtung eines Spenderegisters in Zeiten knapper Ressourcen im deutschen Gesundheitswesen kaum zu rechtfertigen.

Die Sektionen «Sicherheit in der Hämotherapie» und «Präparative und therapeutische Hämapherese» verabschieden deshalb folgendes Votum:

Wir schlagen dringend zunächst eine Datenerhebung durch transfusionsmedizinische Einrichtungen zum Thema «kritische Spenderwanderungen und risikoreiches Spenderverhalten» vor, bevor gesetzliche oder behördliche Vorgaben in irgendeiner Weise beschlossen werden, die unweigerlich zu erheblichen Zusatzkosten führen würden. Vor Einführung ge-

\section{KARGER}

Fax +49 7614520714

(C) 2004 S. Karger GmbH, Freiburg

E-mail Information@Karger.de www.karger.com/tmh

www.karger.com 
setzlicher Grundlagen bzw. behördlicher Überwachungen des Spenderverhaltens sollte insbesondere in Zeiten knapper Finanzmittel zunächst von medizinischer Seite geprüft werden, ob kritische bzw. risikoreiche Spenderwanderungen zwischen Spendeinrichtungen überhaupt bestehen. Anschließend befürworten wir eine Veröffentlichung der Daten und eine Bewertung der Erhebung im Fachgremium (z.B. im Arbeitskreis Blut) bezüglich der etwaigen Notwendigkeit einer Dauerdatenmeldung an Behörden. Das Konzept aus Suhl sollte auf praktische Durchführbarkeit und Routineeinsatz geprüft werden, um eine gemeinsame Lösung zu erarbeiten, die dem Datenschutz gerecht wird und kostenarm anzuwenden ist. Eine Pilotteilnahme möglichst repräsentativer Spende-Einrichtungen ist anzustreben.

Im Namen der Sektionen «Sicherheit in der Hämotherapie» und «Präparative und therapeutische Hämapherese»

\section{Baumann, Münster \\ U. Cassens, Münster G.A. Fabian, Suhl \\ V. Lenz, Heidelberg \\ J. Zingsem, Erlangen}

(Veröffentlichung durch DGTI-Vorstand autorisiert am 3. Mai 2004) 